\title{
Rare earth interstitial-complexes in Ge: hybrid density functional studies
}

\author{
E. Igumbor ${ }^{a, b, *}$ E. Omotoso ${ }^{a}$, H. T. Danga ${ }^{a}$, S. M. Tunhuma ${ }^{a}$, W. E. \\ Meyer $^{\mathrm{a}, * *}$ \\ ${ }^{a}$ Department of Physics, University of Pretoria, Pretoria 0002, South Africa. \\ ${ }^{b}$ Department of Mathematics and Physical Sciences, Samuel Adegboyega University, Km \\ 1 Ogwa/Ehor Rd, Ogwa Edo State Nigeria.
}

\section{Abstract}

We present results of the structural, energetic and electronic properties of rare earth (RE) interstitial-complexes in $\mathrm{Ge}\left(\mathrm{RE}_{\mathrm{Ge}} \mathrm{Ge}_{\mathrm{i}}\right.$; for $\mathrm{RE}$ : Ce, $\mathrm{Pr}, \mathrm{Eu}$, Er and Tm). We used the Heyd, Scuseria, and Ernzerhof (HSE06) hybrid functional within the framework of density functional theory for all calculations. The energy of formation and charge state transition levels of $\mathrm{RE}_{\mathrm{Ge}} \mathrm{Ge}_{\mathrm{i}}$ complexes were obtained. For the neutral charge state, the results of the formation energy of the $\mathrm{RE}_{\mathrm{Ge}} \mathrm{Ge}_{\mathrm{i}}$, were between 0.21 and $8.14 \mathrm{eV}$. Amongst the $\mathrm{RE}_{\mathrm{Ge}} \mathrm{Ge}_{\mathrm{i}}$, while the $\mathrm{Ce}_{\mathrm{Ge}} \mathrm{Ge}_{\mathrm{i}}$ was energetically the most favourable with a binding energy of $3.90 \mathrm{eV}, \mathrm{Tm}_{\mathrm{Ge}} \mathrm{Ge}_{\mathrm{i}}$ and $\mathrm{Er}_{\mathrm{Ge}} \mathrm{Ge}_{\mathrm{i}}$ were not stable with respect to their binding energies. The $\mathrm{Ce}_{\mathrm{Ge}} \mathrm{Ge}_{\mathrm{i}}$ induced deep donor level with negative- $\mathrm{U}$ ordering, the $\mathrm{Pr}_{\mathrm{Ge}} \mathrm{Ge}_{\mathrm{i}}$ induced shallow levels close to the valence band maximum and the $\mathrm{Eu}_{\mathrm{Ge}} \mathrm{Ge}_{\mathrm{i}}$ induced a shallow single donor level.

Keywords: Defects, formation energy, charge state, rare earth

\footnotetext{
${ }^{*}$ Corresponding author

** Corresponding author

Email addresses: elgumuk@gmail.com (E. Igumbor), wmeyer@up.ac.za ( W. E. Meyer )
} 


\section{Introduction}

Rare earth (RE) related defects in semiconductor materials are known for light emission. For example, light emission has been attributed to thulium and erbium defects in material $[1,2]$. The RE elements which have a partially filled inner $4 f$ shell give rise to sharp transitions that are largely insensitive to the crystal host and temperature variations $[1,2]$. Recent reports suggest that electroluminescent behaviour is observed from the optical properties of Tm doped materials [3, 4, 5]. RE substitutional, interstitials and vacancy-complex related defects in $\mathrm{Si}$ and Ge have been studied with emphasis on the induced defect levels $[6,7,8,9,10]$. Ge has a narrow band gap of $0.78 \mathrm{eV}$ at $0 \mathrm{~K}$, and is being considered as a suitable material for next generation high performance microelectronics devices $[11,12,13]$ : such as mobility-enhanced metal-oxide-semiconductor field-effect transistors (MOSFETs). In addition, Ge provides an alternative solution for the search of materials that required high mobility channels. An understanding of defect formation and electrical levels in Ge is essential for the engineering of new Ge-based MOSFETs. For several years, studies on defects in Ge have been attracting attention $[6,7,12]$. A number of RE defects in Ge especially $\operatorname{Tm}[6,7]$ induce negative $-U$ ordering and its vacancy-complexes show charge state controlled metastability.

To provide theoretical insight for the experimental studies of activities of defect levels induced by RE interstitial-complexes in Ge, detailed mod-

elling is essential. In this report, we present results of ab inito study of RE interstitial-complexes of Ge using the Heyd, Scuseria, and Ernzerhof 
(HSE06) hybrid functional [14] within the framework of density functional

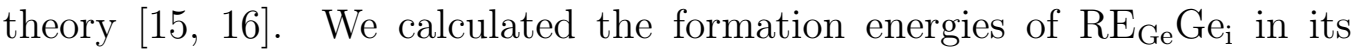
charge states. The induced defect levels of the $\mathrm{RE}_{\mathrm{Ge}} \mathrm{Ge}_{\mathrm{i}}$ were examined by calculating the charge state thermodynamic transition levels.

\section{Computational details}

Density functional theory (DFT) electronic structure calculations using the Vienna Ab initio Simulation Package (VASP) $[17,18]$ have been performed. The core electrons were separated from the valence electrons by using the Projector-augmented wave (PAW) method as implemented in the VASP $[17,19]$. All calculations were carried out using the Heyd, Scuseria, and Ernzerhof (HSE06) [14] hybrid functional. In this hybrid approach, the short-range exchange potential is calculated by mixing a 25 percent fraction of nonlocal Hartree-Fock exchange with the generalized gradient approximation (GGA) functional of Perdew, Burke, and Ernzerhof (PBE) [20]. The hybrid functionals with DFT have been known to accurately predict band gaps of several materials $[21,22,23]$ which the local density approximation (LDA) and the GGA fail to estimate accurately [21, 24, 25]. In addition, the HSE06 has been used to predict accurate band structures and effective masses for InP, InAs, and InSb, where the results are in agreement with experimental data [26]. Recently, Deák et al [27] has shown that tunning the mixing and adjustable parameters of the HSE06 give more accurate predictions of electrical level induced in a wide band gap semiconductor. Although, the HSE06 is efficient for predicting accurately the properties of material, but it is computationally demanding since it requires huge computational resources. 
In the past, the study and prediction of the electronic properties of material with the $f$ orbital valence shell was difficult, because the $f$ orbital is highly localized. Recently, the hybrid functional has been successfully used to predict the electronic and band gap properties of several materials with the $f$ orbital in the valence shell $[7,28,29]$. Following this success of the hybrid functional, it became feasible to handle the $f$ orbital in the valence shell of RE. For the bulk, geometric optimization of Ge was performed using an 8-atom unit cell with an $8^{3}$ Monkhorst-Pack [30] k-point Brillouin zone sampling scheme and cut-off energy of $400 \mathrm{eV}$. A 64 atom supercell was adopted for all calculations. For the defects, Ge atom was introduced in an interstitial site and another Ge atom was replaced with a corresponding RE atom. For the pristine and defect calculations, we used a $2^{3}$ Monkhorst-Pack [30] k-point grid to sample the Brillouin zone. We set the plane wave cut-off of the wave function expansion to $400 \mathrm{eV}$, and refined the geometry until the final change in the total energy and forces were less than $10^{-5} \mathrm{eV}$ and $0.001 \mathrm{eV} / \AA$, respectively. For all calculations, spin orbit coupling was taken into account. In order to calculate the defect formation and thermodynamic charge state transition energy $\left(\epsilon\left(q / q^{\prime}\right)\right)$ levels, the total energy $\mathrm{E}\left(\mathrm{RE}_{\mathrm{Ge}_{\mathrm{e}}} \mathrm{Ge}_{\mathrm{i}}, q\right)$ for a supercell containing the optimized defect $\mathrm{RE}_{\mathrm{Ge}_{\mathrm{e}}} \mathrm{Ge}_{\mathrm{i}}$ in its charge state $q$ was obtained. The defect formation energy $E^{f}\left(\mathrm{RE}_{\mathrm{Ge}} \mathrm{Ge}_{\mathrm{i}}, q\right)$ as a function of electron Fermi energy $\left(\varepsilon_{F}\right)$ is given as $[6,31]$

$E^{f}\left(\mathrm{RE}_{\mathrm{Ge}_{\mathrm{e}}} \mathrm{Ge}_{\mathrm{i}}, q\right)=E\left(\mathrm{RE}_{\mathrm{Ge}} \mathrm{Ge}_{\mathrm{i}}, q\right)-E($ pristine $)+\sum_{i}(\triangle n)_{i} \mu_{i}+q \mu_{e}+E_{c o r}^{q}$

where $E$ (pristine) is the energy of the non-defect supercell, $(\triangle n)_{i}$ is the difference in the number of constituent atoms of type $i$ between the pristine 
and the supercell containing the defect. The chemical potential of different constituent atoms is represented as $\mu_{i}$. The electron chemical potential $\mu_{e}$ is defined as

$$
\mu_{e}=E_{V}+\varepsilon_{F}
$$

where $E_{V}$ is the energy of valence band maximum (VBM). The $\varepsilon_{F}$ is the Fermi energy, which is varied from the VBM to the conduction band minimum (CBM). The correction term $E_{c o r}^{q}$ according to Freysoldt et al [32] was included to account for the shortfall surrounding the calculation of defect formation energies due to errors from the finite-size effects within the supercell and electrostatic potential. A defect transition energy level $\epsilon\left(q / q^{\prime}\right)$ is the Fermi energy at which two charge states $\left(q\right.$ and $\left.q^{\prime}\right)$ of the same defect have the same energy of formation, and is given as $[7,31,32]$

$$
\epsilon\left(q / q^{\prime}\right)=\frac{E^{f}\left(\mathrm{RE}_{\mathrm{Ge}} \mathrm{Ge}_{\mathrm{i}}, q ; \varepsilon_{F}=0\right)-E^{f}\left(\mathrm{RE}_{\mathrm{Ge}} \mathrm{Ge}_{\mathrm{i}}, q^{\prime} ; \varepsilon_{F}=0\right)}{q^{\prime}-q}
$$

Defect-complexes are found to be either stable or unstable (dissociates into non-interacting defects) depending on their binding energies. The binding energy $E_{b}$ which is the energy required to split up a defect-complex into well separated and non-interacting defects is given as $[6,12,33]$

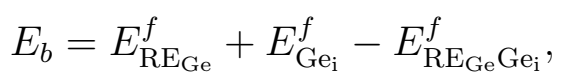

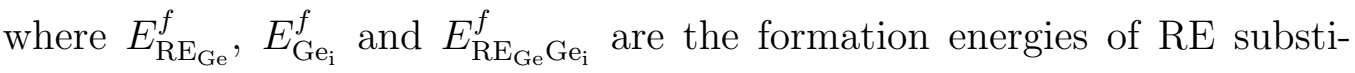
tution in Ge, Ge self-interstitial and RE interstitial-complex of Ge, respectively. Eq. 4 is interpreted as the energy released by the bonded RE interstitial-complex when formed from isolated $\mathrm{Ge}_{\mathrm{i}}$ and $\mathrm{RE}_{\mathrm{Ge}}$. If the binding energy of a $\mathrm{RE}_{\mathrm{Ge}} \mathrm{Ge}_{\mathrm{i}}$ is positive, then the $\mathrm{RE}_{\mathrm{Ge}} \mathrm{Ge}_{\mathrm{i}}$ is stable or otherwise 
unstable. In this present calculation, as reported in Ref [23], we used a modelled band gap of pristine Ge at $0 \mathrm{~K}$ to be $0.78 \mathrm{eV}$.

\section{Results and Discussion}

\subsection{Structural properties}

Fig. 1 displays the relaxed geometric structures of the $\mathrm{RE}_{\mathrm{Ge}} \mathrm{Ge}_{\mathrm{i}}$. After structural relaxation, we found the bond length between Ge-Ge and the bond angle formed between three Ge atoms to be $2.46 \AA$ and $109.40^{\circ}$, respectively. These results are in close agreement with earlier reported results $[34,12]$. For the $\mathrm{Ce}_{\mathrm{Ge}} \mathrm{Ge}_{\mathrm{i}}$ as shown in Fig. 1a, after structural relaxation, the bond distance between a Ce atom and the nearest neighbour Ge atom is $3.11 \AA$, which is $0.05 \AA$ higher than before structural relaxation. Fig. 1b displays the relaxed geometric structure of the $\operatorname{Pr}_{G_{e}} G_{i}$. We observed that the bond angle formed between a Pr atom and its two nearest neighbour Ge counterparts is $110.70^{\circ}$. The bond length formed between Pr and Ge atoms after structural relaxation is $3.35 \AA$, which is about $0.89 \AA$ higher than that of $\mathrm{Ce}-\mathrm{Ge}$. The relaxed geometric structure of the $\mathrm{Eu}_{\mathrm{Ge}} \mathrm{Ge}_{\mathrm{i}}$ is shown in Fig. 1c, the bond distance between an $\mathrm{Eu}$ and its nearest neighbour $\mathrm{Ge}$ atom after(before) structural relation is $2.45(2.45) \AA$, and the angle formed by $\mathrm{Ge}-\mathrm{Eu}-\mathrm{Ge}$ after relaxation is $109.47^{\circ}$. Fig. 1d displays the relaxed ge-

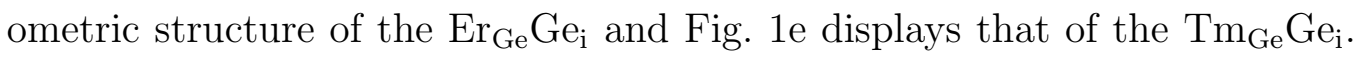
For the Er, Tm and their nearest neighbours Ge atoms, the bond distance between them after(before) structural relation is $2.75(2.45)$ and $3.21(2.45) \AA$, respectively. The Er-Ge and Tm-Ge bond length are 0.29 and 0.75 , respectively, higher than that of the Ge-Ge. Ge-Tm-Ge and Ge-Er-Ge bond angles 
are $111.99^{\circ}$ and $105.32^{\circ}$, respectively. While the bond length between the Ge interstitial atom and the RE substitutional impurity, remain the same before and after structural relaxation for the $\mathrm{Eu}$, Er and Tm, for the Ce and Pr there is an increase of the bond length by 0.54 and $0.26 \AA$, respectively. The covalent radii between a Ge atom and a $\mathrm{RE}$ atom is 3.07, 2.79, 2.78, 2.87 and $2.87 \AA$ for the Eu, Er, Tm, Ce and Pr respectively. Amongst the $\mathrm{RE}_{\mathrm{Ge}} \mathrm{Ge}_{\mathrm{i}}$, the $\mathrm{Er}_{\mathrm{Ge}} \mathrm{Ge}_{\mathrm{i}}$ and $\mathrm{Tm}_{\mathrm{Ge}} \mathrm{Ge}_{\mathrm{i}}$, experience more strain in theei bond lengths than the others. The amount of strain experienced by the various $\mathrm{RE}_{\mathrm{Ge}} \mathrm{Ge}_{\mathrm{i}}$, plays vital role in predicting the formation energies and the most energetically favourable defect-complex.

\subsection{Electronic properties}

Fig. 2 displays plot of the projected density of states (PDOS) and total density of states (DOS) for both the pristine Ge and $\mathrm{RE}_{\mathrm{Ge}} \mathrm{Ge}_{\mathrm{i}}$. The plot of PDOS in Fig. 2a for the pristine Ge shows that the minority and majority spins are symmetrically the same, and hence the system is not spin polarised. According to Fig. $2 \mathrm{~b}$, the $\mathrm{Ce}_{\mathrm{Ge}} \mathrm{Ge}_{\mathrm{i}}$ induced orbital states inside the band gap of Ge, leading to a metallic system. The majority and minority spins of

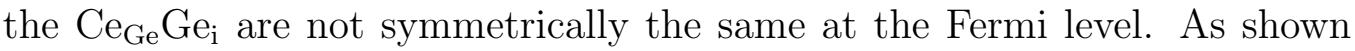
in Fig. 2c, for the $\mathrm{Er}_{\mathrm{Ge}} \mathrm{Ge}_{\mathrm{i}}$, the defect introduced orbital states inside the band gap, which are $0.27 \mathrm{eV}$ below the Fermi level at the conduction band minimum. This suggests that the $\mathrm{Er}_{\mathrm{Ge}} \mathrm{Ge}_{i}$ posses a semi-metallic character. For the $\mathrm{Eu}_{\mathrm{Ge}} \mathrm{Ge}_{\mathrm{i}}$ see (Fig. 2d) we found the ground states of this system at the conduction band to induced orbital states at 0.29 and $0.06 \mathrm{eV}$ below the Fermi level for the spin up and spin down, respectively. These energy levels show that the $\mathrm{Eu}$ is spin polarised. The $\operatorname{Pr}_{\mathrm{Ge}} \mathrm{Ge}_{\mathrm{i}}$ and $\mathrm{Tm}_{\mathrm{Ge}} \mathrm{Ge}_{\mathrm{i}}$ displayed in 
Figs. 2e and 2f, respectively, exhibit strong metallic character in addition to the spin dependency of the system. For all $\mathrm{RE}_{\mathrm{Ge}} \mathrm{Ge}_{\mathrm{i}}$, we observed the effect of strong orbital hybridization between the $p$ orbital of both RE and Ge atoms. Noticeably ground state orbital hybridization between $s$ and $p$ orbitals of Ge and $\mathrm{RE}$ atoms, respectively are observed for all $\mathrm{RE}_{\mathrm{Ge}} \mathrm{Ge}_{\mathrm{i}}$. In addition, strong $d$ orbital hybridization was observed. Except for the $\mathrm{Ce}_{\mathrm{Ge}} \mathrm{Ge}_{\mathrm{i}}$, the ground state $d$ orbital of the $\mathrm{RE}_{\mathrm{Ge}} \mathrm{Ge}_{\mathrm{i}}$ which is located below the Fermi level (at the CBM) for the Er and Eu, or above the Fermi level (at the VBM) for the Tm and $\mathrm{P}$ contributed to the states in the band gap of Ge as shown by Fig. 2 .

\subsection{Formation energy and thermodynamic defect levels of $R E_{\mathrm{Ge}} G e_{\mathrm{i}}$}

Table 1 lists formation and binding energies for the neutral charge state of $\mathrm{RE}_{\mathrm{Ge}} \mathrm{Ge}_{\mathrm{i}}$. Table 2 lists charge state thermodynamic transition energy levels induced by $\mathrm{RE}_{\mathrm{Ge}} \mathrm{Ge}_{\mathrm{i}}$. Fig. 3 displays plot of formation energy as a function of the Fermi energy for $\mathrm{RE}_{\mathrm{Ge}} \mathrm{Ge}_{\mathrm{i}}$. The defect-complex of $\mathrm{Ce}_{\mathrm{Ge}} \mathrm{Ge}_{\mathrm{i}}$ has an energy of formation of $0.21 \mathrm{eV}$ for the neutral charge state and a binding energy of $3.90 \mathrm{eV}$. These results suggest that the defect $\mathrm{Ce}_{\mathrm{Ge}} \mathrm{Ge}_{\mathrm{i}}$ is stable and its dissociation into non-interacting defects will occur at the expense of energy. The $\mathrm{Ce}_{\mathrm{Ge}} \mathrm{Ge}_{\mathrm{i}}$ induced a deep level charge state transition at $(+1 /-1)$ with energy of $0.49 \mathrm{eV}$ above the VBM (see Fig. 3a). Another charge state transition level induced by the $\mathrm{Ce}_{\mathrm{Ge}} \mathrm{Ge}_{\mathrm{i}}$, although accessible but not thermodynamically stable is the $(+2 /-1)$, which is at least $0.12 \mathrm{eV}$ away from the thermodynamically stable region. Negative-U charge state ordering occurs in a defect where the neutral charge state is excited, this can be primarily caused by a large lattice distortion. The $(+1 /-1)$ transition level induced by the $\mathrm{Ce}_{\mathrm{Ge}} \mathrm{Ge}_{\mathrm{i}}$ exhibits a negative-U ordering. The formation energy of the 
$\operatorname{Pr}_{\mathrm{Ge}} \mathrm{Ge}_{\mathrm{i}}$ is $3.21 \mathrm{eV}$, which is $3.00 \mathrm{eV}$ higher than that of the $\mathrm{Ce}_{\mathrm{Ge}} \mathrm{Ge}_{\mathrm{i}} \cdot \operatorname{Pr}_{\mathrm{Ge}} \mathrm{Ge}_{\mathrm{i}}$ has a binding energy of $0.75 \mathrm{eV}$, which suggests that under equilibrium condition the defect is stable. The calculated charge state transition levels of the $\operatorname{Pr}_{\mathrm{Ge}} \mathrm{Ge}_{\mathrm{i}}$ as shown in Fig. 3b are lying close to the band edges. A shallow

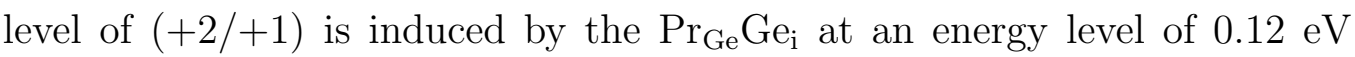
above the VBM. The $\operatorname{Pr}_{\mathrm{Ge}} \mathrm{Ge}_{\mathrm{i}}$ exhibits properties of negative-U ordering.

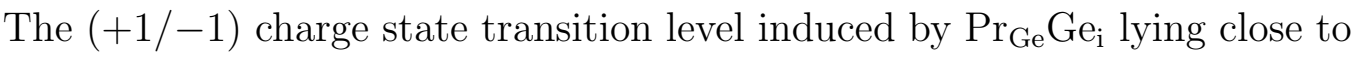
the CBM with energy $0.01 \mathrm{eV}$ is a negative-U. There are other charge state

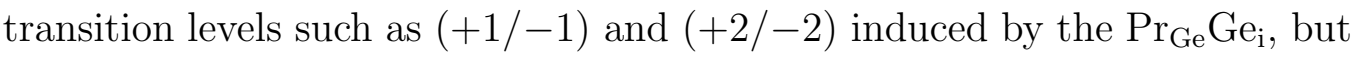

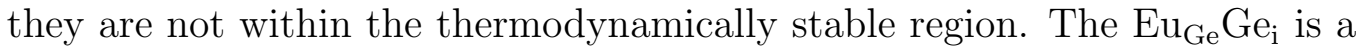
stable defect-complex system with a binding energy of $1.25 \mathrm{eV}$. The $\mathrm{Eu}_{\mathrm{Ge}} \mathrm{Ge}_{\mathrm{i}}$ induced a shallow single donor level at $\mathrm{E}_{\mathrm{C}}+0.12$. Other notable transition levels induced by the $\mathrm{Eu}_{\mathrm{Ge}} \mathrm{Ge}_{\mathrm{i}}$ are not within the thermodynamically stable region.

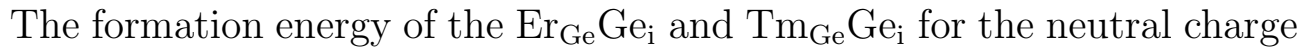
state are 8.14 and $6.54 \mathrm{eV}$, respectively. $\mathrm{Er}_{\mathrm{Ge}} \mathrm{Ge}_{\mathrm{i}}$ and $\mathrm{Tm}_{\mathrm{Ge}} \mathrm{Ge}_{\mathrm{i}}$ have binding energies of -0.91 and $-0.54 \mathrm{eV}$, respectively. These energies according to Eq. 4, suggest that both defect-complex systems can easily dissociate into non-interacting defects. The implication is that the $\operatorname{Er}_{G e} G_{i}$ and $\operatorname{Tm}_{G e} G_{i}$ are not stable. Since these defect-complex systems are not stable, we did not investigate further the activities of their defect induced levels.

\section{Summary}

By using the HSE06 hybrid functional with DFT, we performed electronic structure calculation of rare earth interstitial complex in $\mathrm{Ge}\left(\mathrm{RE}_{\mathrm{Ge}} \mathrm{Ge}_{\mathrm{i}}\right)$. Fur- 
thermore, we calculated the energy of formation and charge state transition levels of the $\mathrm{RE}_{\mathrm{Ge}} \mathrm{Ge}_{\mathrm{i}}$. The $\mathrm{Ce}_{\mathrm{Ge}} \mathrm{Ge}_{\mathrm{i}}$ was found to be the most stable complex with a binding energy of $3.90 \mathrm{eV}$. Amongst the $\mathrm{RE}_{\mathrm{Ge}} \mathrm{Ge}_{\mathrm{i}}, \mathrm{Ce}_{\mathrm{Ge}} \mathrm{Ge}_{\mathrm{i}}$ was

found to be energetically the most favourable with a formation energy of $0.21 \mathrm{eV}$. The $\mathrm{Tm}_{\mathrm{Ge}} \mathrm{Ge}_{\mathrm{i}}$ and $\mathrm{Er}_{\mathrm{Ge}} \mathrm{Ge}_{\mathrm{i}}$ were unstable and have the tendency to disintegrate into non-interacting defects. The $\mathrm{Ce}_{\mathrm{Ge}} \mathrm{Ge}_{\mathrm{i}}, \operatorname{Pr}_{\mathrm{Ge}} \mathrm{Ge}_{\mathrm{i}}$ and $\mathrm{Er}_{\mathrm{Ge}} \mathrm{Ge}_{\mathrm{i}}$ induced charge state transition levels within the band gap of Ge. In addition, the $\mathrm{Ce}_{\mathrm{Ge}} \mathrm{Ge}_{\mathrm{i}}$ and $\mathrm{Pr}_{\mathrm{Ge}} \mathrm{Ge}_{\mathrm{i}}$ exhibit properties of negative- $\mathrm{U}$ ordering. The information we have presented in this report should act as a frontier insight for experiment synthesis of the $\mathrm{RE}_{\mathrm{Ge}} \mathrm{Ge}_{\mathrm{i}}$. The authors acknowledge MedeA.

\section{Acknowledgement}

This work is based on the research supported partly by National Research foundation (NRF) of South Africa (Grant specific unique reference number (UID) 98961). The opinions, findings and conclusion expressed are those of the authors and the NRF accepts no liability whatsoever in this regard.

[1] M. Lourenço, C. Opoku, R. Gwilliam, K. Homewood, Optical Materials 32(12), 1597 (2010)

[2] A. Polman, Journal of Applied Physics 82(1), 1 (1997)

[3] F. Fang, A. Ng, X. Chen, A. Djurii, Y. Zhong, K. Wong, P. Fong, H. Lui, C. Surya, W. Chan, Materials Chemistry and Physics 125(3), 813 (2011)

[4] M. Peres, J. Wang, M. Soares, A. Neves, T. Monteiro, E. Rita, U. Wahl, J. Correia, E. Alves, Superlattices and Microstructures 36(46), 747 (2004) 
[5] T. Monteiro, A.J. Neves, M.J. Soares, M.C. Carmo, M. Peres, E. Alves, E. Rita, Applied Physics Letters 87(19), 192108 (2005)

[6] E. Igumbor, R.E. Mapasha, R. Andrew, W.E. Meyer, Computational Condensed Matter 8, 31 (2016)

[7] E. Igumbor, W. Meyer, Materials Science in Semiconductor Processing 43, $129(2016)$

[8] R. Jones, Optical Materials 28(6), 718 (2006)

[9] F.D. Auret, S.M.M. Coelho, M. Hayes, W.E. Meyer, J.M. Nel, physica status solidi (a) $\mathbf{2 0 5 ( 1 ) , ~} 159$ (2008)

[10] E. Simoen, J. Vanhellemont, Journal of Applied Physics 106(10), 103516 (2009)

[11] C. Claeys, E. Simoen, Germanium-based technologies: from materials to devices (Elsevier, 2011)

[12] E. Igumbor, R.E. Mapasha, W.E. Meyer, Journal of Electronic Materials pp. 1-8 (2016). URL http://dx.doi.org/10.1007/s11664-016-5026-z

[13] E. Igumbor, R.C. Andrew, W.E. Meyer, Journal of Electronic Materials 46(2), $1022(2017)$

[14] J. Heyd, G.E. Scuseria, M. Ernzerhof, The Journal of Chemical Physics 118(18), 8207 (2003)

[15] P. Hohenberg, W. Kohn, Phys. Rev. 136, B864 (1964)

[16] W. Kohn, Journal of computational chemistry 20(1), 1 (1999) 
[17] G. Kresse, J. Furthmüller, Phys. Rev. B 54, 11169 (1996)

[18] G. Kresse, D. Joubert, Phys. Rev. B 59, 1758 (1999)

[19] P.E. Blochl, Phys. Rev. B 50, 17953 (1994)

[20] J.P. Perdew, K. Burke, M. Ernzerhof, Phys. Rev. Lett. 77, 3865 (1996)

[21] H. Tahini, A. Chroneos, R.W. Grimes, U. Schwingenschlgl, H. Bracht, Applied Physics Letters 99(7), 072112 (2011)

[22] P. Deák, B. Aradi, T. Frauenheim, E. Janzén, A. Gali, Phys. Rev. B 81, $153203(2010)$

[23] E. Igumbor, C. Ouma, G. Webb, W. Meyer, Physica B: Condensed Matter 480, 191 (2016)

[24] P. Śpiewak, J. Vanhellemont, K. Sueoka, K. Kurzydłowski, I. Romandic, Materials Science in Semiconductor Processing 11(5), 328 (2008)

[25] E. Igumbor, K. Obodo, W.E. Meyer, Solid State Phenomena 242, 440 (2015)

[26] Y.S. Kim, K. Hummer, G. Kresse, Physical Review B 80(3), 035203 (2009)

[27] P. Deák, Q.D. Ho, F. Seemann, B. Aradi, M. Lorke, T. Frauenheim, Physical Review B 95(7), 075208 (2017)

[28] B.G. Janesko, T.M. Henderson, G.E. Scuseria, Physical Chemistry Chemical Physics 11(3), 443 (2009) 
[29] J.L. Da Silva, M.V. Ganduglia-Pirovano, J. Sauer, V. Bayer, G. Kresse, Physical Review B 75(4), 045121 (2007)

[30] H.J. Monkhorst, J.D. Pack, Phys. Rev. B 13, 5188 (1976)

[31] Y. Kumagai, F. Oba, Phys. Rev. B 89, 195205 (2014)

[32] C. Freysoldt, J. Neugebauer, C.G. Van de Walle, physica status solidi (b) 248(5), 1067 (2011)

[33] G. Zollo, Y.J. Lee, R.M. Nieminen, Journal of Physics: Condensed Matter 16(49), 8991 (2004)

[34] A. Chroneos, B.P. Uberuaga, R.W. Grimes, Journal of Applied Physics 102(8), 083707 (2007)

Table 1: The energy of formation $E^{f}\left(R E_{G e} G e_{i}, q\right)$ and binding energies $\left(E_{b}\right)$ of $\mathrm{RE}_{\mathrm{Ge}_{\mathrm{e}} \mathrm{Ge}_{\mathrm{i}}}$ complexes.

\begin{tabular}{cccccc}
\hline & $\mathrm{Ce}_{\mathrm{Ge}_{\mathrm{G}} \mathrm{Ge}_{\mathrm{i}}}$ & $\mathrm{Pr}_{\mathrm{Ge}} \mathrm{Ge}_{\mathrm{i}}$ & $\mathrm{Eu}_{\mathrm{Ge}} \mathrm{Ge}_{\mathrm{i}}$ & $\mathrm{Er}_{\mathrm{Ge}} \mathrm{Ge}_{\mathrm{i}}$ & $\mathrm{Tm}_{\mathrm{Ge}} \mathrm{Ge}_{\mathrm{i}}$ \\
\hline$E^{f}\left(\mathrm{RE}_{\mathrm{Ge}} \mathrm{Ge}_{\mathrm{i}}, q\right)(\mathrm{eV})$ & 0.21 & 3.21 & 2.64 & 8.14 & 6.54 \\
$E_{b}(\mathrm{eV})$ & 3.90 & 0.75 & 1.25 & -0.91 & -0.54 \\
\hline
\end{tabular}

Table 2: The charge state transition energy levels $\epsilon\left(q / q^{\prime}\right)$ of the stable $\mathrm{RE}_{\mathrm{Ge}} \mathrm{Ge}_{\mathrm{i}}$ within the band gap of Ge. These energy levels were calculated with respect to the VBM and measured in $\mathrm{eV}$.

\begin{tabular}{cccc} 
Charge state transition level & $\mathrm{Ce}$ & $\mathrm{Pr}$ & $\mathrm{Eu}$ \\
\hline$(+2 /+1)$ & - & 0.12 & - \\
$(+1 / 0)$ & - & - & 0.66 \\
$(+1 /-1)$ & 0.49 & 0.77 & - \\
\hline
\end{tabular}




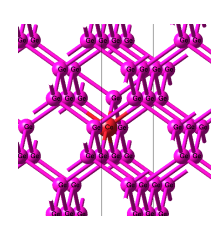

(a)

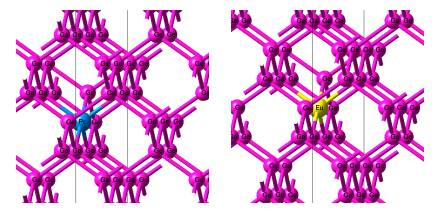

(b) (c)

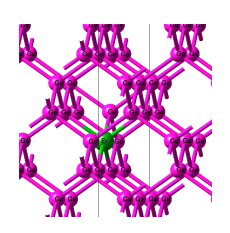

(d)

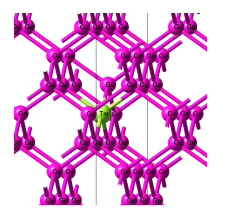

(e)

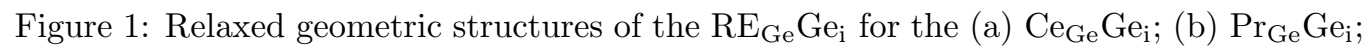
(c) $\mathrm{Eu}_{\mathrm{Ge}} \mathrm{Ge}_{\mathrm{i}} ;$ (d) $\mathrm{Er}_{\mathrm{Ge}} \mathrm{Ge}_{\mathrm{i}}$ and (e) $\mathrm{Tm}_{\mathrm{Ge}} \mathrm{Ge}_{\mathrm{i}}$.

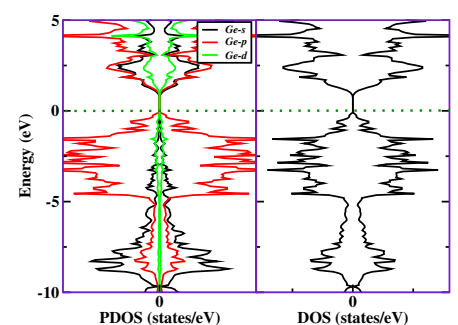

(a)

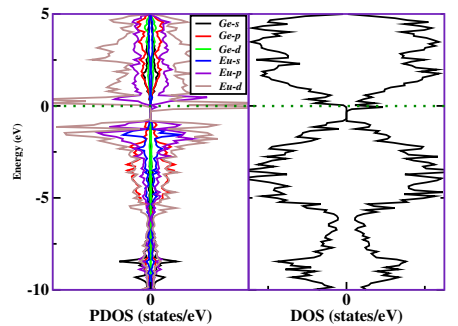

(d)

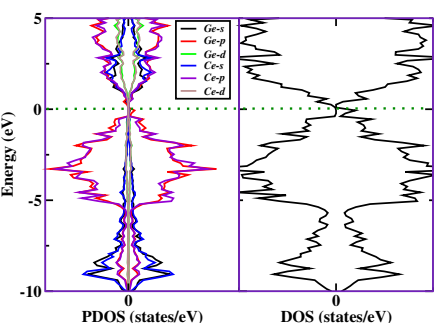

(b)

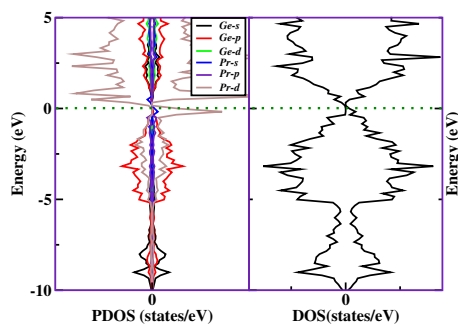

(e)

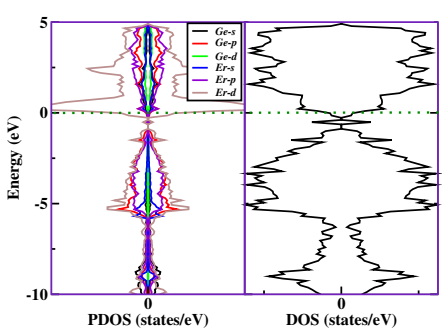

(c)

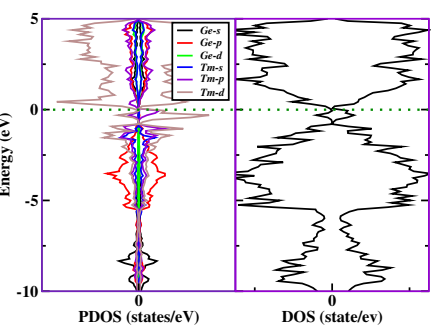

(f)

Figure 2: Plots of projected density of states (PDOS) and total density of states (DOS) at $\varepsilon_{F}=0$ for the $\mathrm{RE}_{\mathrm{Ge}} \mathrm{Ge}_{\mathrm{i}}$ complexes. The green dash line is the Fermi level; (a) Pristine Ge (b) $\mathrm{Ce}_{\mathrm{Ge}} \mathrm{Ge}_{\mathrm{i}}$; (c) $\mathrm{Eu}_{\mathrm{Ge}} \mathrm{Ge}_{\mathrm{i}}$; (d) $\mathrm{Er}_{\mathrm{Ge}} \mathrm{Ge}_{\mathrm{i}}$; (e) $\operatorname{Pr}_{\mathrm{Ge}} \mathrm{Ge}_{\mathrm{i}}$ and (f) $\mathrm{Tm}_{\mathrm{Ge}} \mathrm{Ge}_{\mathrm{i}}$. 


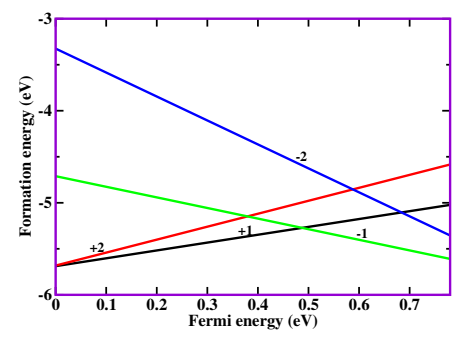

(a)

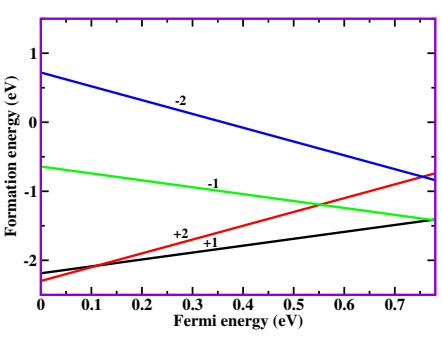

(b)

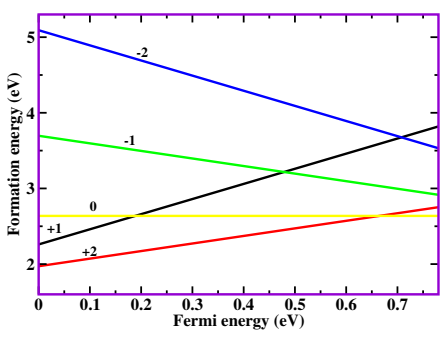

(c)

Figure 3: Plot of formation energy as a function of the Fermi energy of the $\mathrm{RE}_{\mathrm{Ge}} \mathrm{Ge}_{\mathrm{i}}$

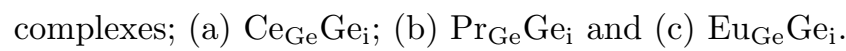

